\section{Optimisation of an automated drusen- quantifying software for the analysis of drusen distribution in patients with age- related macular degeneration}

${ }^{1}$ Oxford Eye Hospital and the University of Oxford, Oxford, UK

${ }^{2}$ Columbia University, New York, NY, USA

${ }^{3}$ King's College Hospital, London, UK

${ }^{4}$ Erasmus University Medical Center, Rotterdam, The Netherlands

Correspondence: BB Ong, Department of Ophthalmology, Oxford Eye Hospital, John Radcliffe Hospital, Oxford OX1 3DU, UK.

Tel: + 44 (0)7917404851.

E-mail: ongbeng2@

doctors.org.uk

Received: 19 July 2012 Accepted in revised form: 30 November 2012 Published online: 11 January 2013

This work has previously been presented at the European Society of Retinal Specialists and World Ophthalmology Congress annual meetings.

\begin{abstract}
Purpose The purpose of this study is to optimise the settings of the Retinal Image Analysis Laboratory (RIALAB), a semiautomatic drusen quantification software, in planning for high-throughput quantification of drusen in clinical studies of age-related macular degeneration (AMD).

Patients and methods A comparison of five different settings in RIALAB was made on 67 images from the Rotterdam eye study (population-based study) and 56 images from the fellow eye of patients with active neovascular AMD in King's College Hospital, London (hospital-based study).

Results The 'Few Outer' setting was the best setting, with it being most appropriate for $52(77.6 \%)$ of the Rotterdam cohort and 47 (83.9\%) for the London cohort. Pearson's $\chi^{2}$-test revealed both results to be statistically significant $(P<0.0001)$.

Conclusions RIALAB is a viable algorithm and software package that can detect, quantify, and analyse drusen efficiently in both population-based and hospital-based studies. We have shown that the 'Few Outer' drusen setting can be employed as the default setting, with fine-tuning only needed in a minority of cases, thus helping to speed up workflow.

Eye (2013) 27, 554-560; doi:10.1038/eye.2012.292; published online 11 January 2013
\end{abstract}

Keywords: age-related macular degeneration; automated drusen delineation; image processing
BB Ong ${ }^{1}$, N Lee², WP Lee ${ }^{1}$, E Pearce ${ }^{3}$ S Sivaprasad ${ }^{3}$, CC Klaver ${ }^{4}$, RT Smith ${ }^{2}$ and NV Chong ${ }^{1}$

\section{Introduction}

Age-related macular degeneration (AMD) is the leading cause of irreversible blindness in the developed world. AMD is a progressive disease with early detectable changes, such as drusen, which are thought to occur as a result of lifetime exposure to oxidative stress. As the macula mediates central photopic vision, macular pathology can result in a significant deterioration of visual acuity.

As drusen seldom affect the photoreceptors directly, patients are usually asymptomatic with drusen. However, the presence of drusen indicates an increased risk of progressive disease towards either advanced dry AMD or choroidal neovascularisation. Studies have shown that the size and extensiveness of drusen distribution influence the risk of progression; ${ }^{1}$ therefore, the need for proper drusen identification in screening programmes is crucial.

The Age-Related Eye Disease Study has found a role for high-dose micronutrient supplementation consisting vitamins $\mathrm{C}, \mathrm{E}$, $\beta$-carotene, and zinc, which is able to bring about a $25 \%$ reduction in 5-year risk of progression to advanced AMD for patients with large or extensive intermediate drusen. ${ }^{1}$

Because of the potentially serious implications of $\mathrm{AMD}$, monitoring its progression remains of paramount importance. Monitoring drusen may help to predict the conversion of dry to wet AMD-the Rotterdam eye study has shown that having more than $10 \%$ 
of macular area covered by drusen conferred a sixfold risk in progression towards more severe AMD over 2 years. $^{2}$ However, having a more reliable method of identifying the progression patterns may help to group people into more precise at-risk groups, especially with the advent of increased knowledge regarding the genetics of AMD.

There has recently been significant interest in the field of drusen segmentation. Previous work has largely focused on strategies utilising localised threshold graphical methods of delineating drusen. ${ }^{3,4}$ More recent research has demonstrated that computer software is able to automatically distinguish between the drusen in AMD from similar-looking pisciform deposits and flecks in Stargardt disease. ${ }^{5}$ Very recent work also has started to shed light on OCT-based systems, but while SD-OCT provides excellent visualisation of drusen-related RPE disease there are still limitations in the automated segmentation algorithms' ability to reliably identify drusen amount and size, especially for smaller drusen. ${ }^{6}$

Current clinical practice involves manual drusen quantification, which has always been labour- and timeintensive, as well as subject to observer error due to the inherent subtle differences that drusen may exhibit. Here we present and test a software prototype called the Retinal Image Analysis Laboratory (RIALAB), which is able to utilise computational processing power to automate the quantification of drusen, using a global thresholding technique known as the Otsu method. RIALAB has multiple settings that can be customised to optimise its output based on the retinal photograph's drusen characteristics.

To further improve its throughput for future use in studying a large number of images and eliminate the need for manual adjustment for each fundus photograph, we performed a cross-validation study to identify if a particular phenotype of drusen was present in the cohort, from which we could optimise the software's algorithm parameters and develop a standard default setting for the software to improve its efficiency.

Furthermore, recent research has started to show that drusen load may have a functional correlation with functional measures (work presented by Pearce $e t a l^{7}$ at the Association of Research in Vision and Ophthalmology Annual Meeting 2012), as well as prognosis. ${ }^{1}$ As such, there emerges a need for a reliable and accurate method that can identify the distribution and quantify the burden of drusen in the eye. Automated software packages such as RIALAB provide such a means, but need to be assessed to see whether it is robust enough to be used in both a hospital and a population setting.

Previous work on automated software has largely revolved around pilot tests on small numbers of patients to assess usability. However, they have largely again been theoretical experiments based on assessing multiple subtypes of AMD phenotypes. This paper aims to identify which drusen subtypes are most relevant in real life by being manifested more often in the population, and to test the software under real-world conditions.

\section{Materials and methods}

For this study, only retinal images of patients who were anonymised and had all identifiable personal data removed were used. This study also conformed to principles of the Declaration of Helsinki for medical research. ${ }^{8}$

For our analysis, we utilised the RIALAB software package, a preliminary research prototype, which has been developed at Heffner Biomedical Imaging Lab. ${ }^{9}$ RIALAB is a graphical platform designed to assist in the viewing, editing, and analysing of retinal images. It is based on a mathematical algorithm first published in $2005,{ }^{10}$ which essentially utilises the techniques of luteal compensation and background levelling to achieve a satisfactory delineation of drusen area. The Otsu method, an automatic histogram-based global thresholding technique, ${ }^{11}$ is first utilised to provide an initial input to the background model for each zone. This initial input is then subjected to a number of iterations of the luteal compensation and background levelling steps of the algorithm above to refine the background model. Finally, the Otsu method is then rerun to obtain a finalised result.

Images (in any one of the common picture formats, such as JPEG or GIF) were loaded onto RIALAB. The user then selects the laterality of the photograph-either the left eye (oculus sinister or 'OS') or right eye (oculus dexter or ' $O D^{\prime}$ ). Then, with the mouse pointer, the centre of the macula (the fovea centralis) and the closest border of the optic disc to the fovea centralis were selected to orient the software. From these initial selections, a $324 \times 324$ pixel image is cropped from the overall photograph to focus subsequent analysis, with each pixel representing approximately $18.5 \mu \mathrm{m}$ of length.

RIALAB then identifies and delineates the drusen, as well as localises them to various segments. The paradigm that is adopted is to visualise the macular area into two circles (the 'two-zone model'): an inner circle and an outer annulus, which are then further subdivided into four quadrants, giving a total of eight areas (Figure 1). RIALAB is then able to quantify the amount of drusen in each quadrant, and exports these to a spreadsheet to enable easy further statistical analysis (Figure 2).

The manual adjustment of RIALAB settings enables improved identification and area delineation of drusen. This is useful as drusen may manifest in various distributions. RIALAB was designed with five 


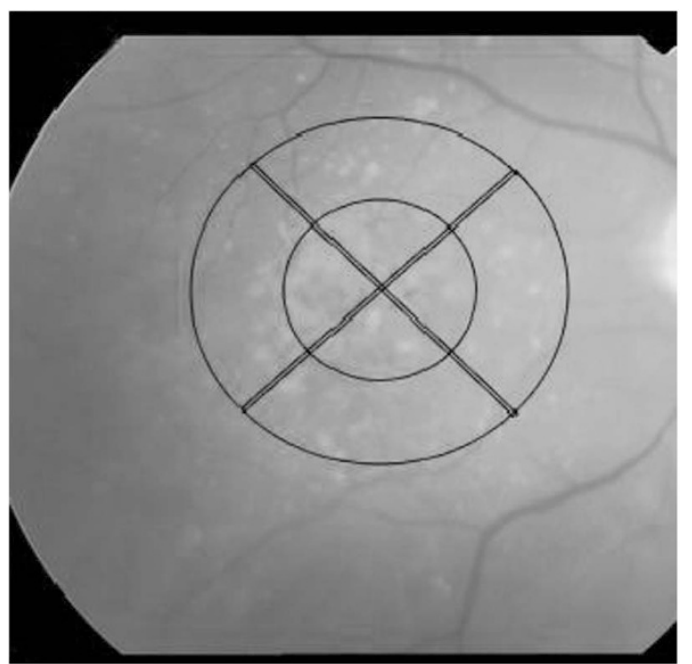

Figure 1 Fundus photograph of the right eye with a conceptualised diagram superimposed on it. The central macular area is divided into an inner circle (3000 $\mu \mathrm{m}$ in radius) and an outer annulus ( $6000 \mu \mathrm{m}$ in radius). The circles are then further subdivided into four quadrants, namely the superior, inferior, temporal, and nasal quadrants, giving a total of eight areas for analysis.

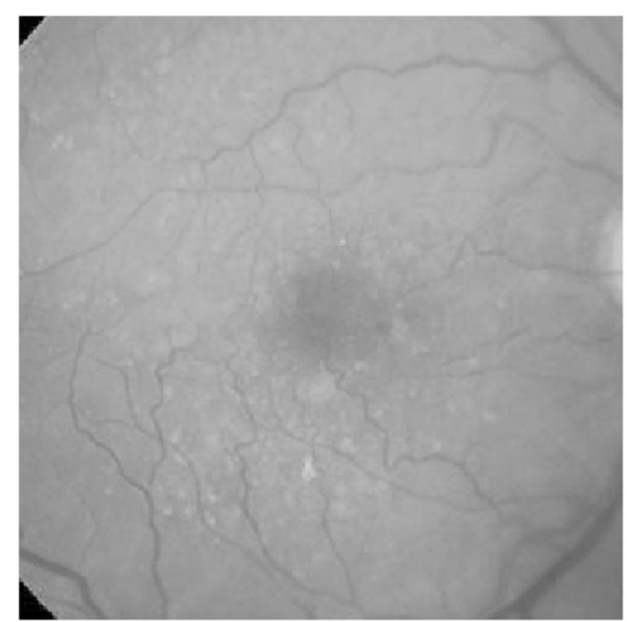

Figure 2 Example of a retina with drusen, and its numerical output (Table 1).

pre-defined setting parameters, which allowed some user-defined customisation of the analyses. Drusen may be 'hard' (exhibit sharp boundaries) or 'soft' (exhibit soft boundaries). They could also be sparsely located in the inner zone ('few inner zone') or the outer zone ('few outer zone'). Furthermore, the presence of pebble-like drusen could be accounted for. From these settings we derived a few combinations of default settings through miniature pilot studies, which had the best fit of drusen empirically, as decided by a consultant ophthalmologist (VC), from which we proceeded to test:

1. Few inner, soft inner (sparse distribution of predominantly soft, large drusen in the inner 3000- $\mu \mathrm{m}$ zone)

2. Few inner (sparse distribution of drusen in the inner 3000- $\mu \mathrm{m}$ zone)

3. Few outer (sparse distribution of drusen in the outer $6000-\mu \mathrm{m}$ annulus)

4. Soft inner (predominantly soft, large drusen in the inner 3000- $\mu \mathrm{m}$ zone)

5. Few inner, soft outer (sparse distribution of drusen in inner 3000- $\mu \mathrm{m}$ zone, with drusen in the outer $6000-\mu \mathrm{m}$ annulus being predominantly soft and large)

We analysed two cohorts of patients using the RIALAB software. The first cohort involved taking a random sample from a population study (the Rotterdam Elderly Study). ${ }^{12,13}$ And the second cohort is the fellow eye of patients with active unilateral neovascular AMD from King's College Hospital, London.

For the Rotterdam cohort, we selected a sample of 200 photographs from the enriched AMD database of 1508 images. The enriched AMD database has images of patients that have at least one eye having signs of AMD, with good image quality. Of these 200 photographs, only $67(33.5 \%)$ had the appearance of early AMD, as evidenced by the presence of drusen. Images that had no drusen and those with end-stage disciform scars were removed.

The Rotterdam Study is a large, prospective, singlecentre population cohort study, first started in 1990, which aims to investigate the epidemiology, aetiology, and progression of illnesses in four broad areasneurogeriatric, cardiovascular, locomotor, and ophthalmic diseases. The entire population over 55 years of age in the district of Ommoord in Rotterdam, The Netherlands, was invited to join the study. Initially, 7983 subjects were recruited when the study was commenced in 1990; in 2002, a further 3011 subjects were added, bringing the overall total to 10994 subjects to date. ${ }^{14}$

The London cohort is from 56 images at the King's College Hospital, London, with known unilateral active neovascular AMD with a relatively preserved fellow eye. The fellow eye was used in the study.

An observer then looked at each of the images and picked the setting that was thought to best represent the drusen graphically for each image. The results were subsequently tabulated in a spreadsheet. To test for reproducibility, the same observer was instructed to reassess the images again 1 week later, and his output the second time around were compared with that from the initial round. To improve validity, another observer was 
also asked to look at the same images and to list her choices as to which of the settings were most suitable, and this was subsequently compared with the first observer's output.

The same process was repeated for this batch of 56 photographs from the hospital study. A Pearson's $\chi^{2}$-test was carried out to check for statistical significance of any findings.

For the purposes of our study, we took the best setting to be the setting that most closely delineates the drusen in the photograph. This was determined by a lay-person who analysed the photographs and determined which setting would best visually match the area of drusen. RIALAB was designed with the hope of being able to provide accessibility to various members of the clinical team, and with this endpoint in mind a lay-person was used as the subject.

\section{Results}

The 'Few Outer' combination setting was the best setting for 77.6 and $83.9 \%$ of the images in the Rotterdam cohort and London cohort, respectively, whereas the Pearson's $\chi^{2}$-test revealed the probability of observing this distribution among the various settings is statistically significant $(P<0.0001)$. The details were summarised in Table 2. An example of the software in action is depicted in Figure 3a. The outputs from the various settings, when run against an example photograph, are shown in Figure 3b. Table 2 and Figure 4 show the results for the Rotterdam Study and London AMD cohorts.

In terms of reproducibility, the same initial observer when asked to reassess the same images 1 week later had a concordance rate of 65 out of 67 images (97.0\%). In addition, the inter-observer concordance rate between the first observer and second observer was 64/67 (95.5\%).

\section{Discussion}

In this study, we have shown that RIALAB is a potentially viable method of detecting drusen automatically, and a default setting of the 'Few Outer' drusen can be selected to further optimise its workflow.

The ability of RIALAB to delineate and quantify the drusen means it would also have potential in obtaining standard and reproducible longitudinal measurements of patients' drusen development, thus facilitating the clinician in assessing the progression of the patient's AMD.

There has been tremendous variability in the way similar studies have sought to confirm the validity of the analyses. For example, Rapantzikos et al ${ }^{15}$ assessed their output against two expert analyses, and the interception between the experts' gradings were used. On the other hand, data from Smith et al ${ }^{10}$ were evaluated by one
Table 1 Example of the numerical output produced by RIALAB from a corresponding photograph

\begin{tabular}{lccccccc}
\hline & $\begin{array}{c}\text { Dr } \\
\text { Area }\end{array}$ & $\begin{array}{c}\text { Small } \\
\text { Dr }\end{array}$ & $\begin{array}{c}\text { Intermed } \\
\text { Dr }\end{array}$ & $\begin{array}{c}\text { Large } \\
\text { Dr }\end{array}$ & $\begin{array}{c}\text { Hypo } \\
\text { area }\end{array}$ & $\begin{array}{c}\text { Brite } \\
\text { lesion }\end{array}$ & $\begin{array}{c}\text { Huge } \\
\text { lesion }\end{array}$ \\
\hline $\begin{array}{l}\text { Inner } \\
\text { circ }\end{array}$ & 180 & 3 & 8 & 4 & No & No & No \\
$\begin{array}{l}\text { Middle } \\
\text { circ } \\
\begin{array}{l}\text { Outer } \\
\text { circ }\end{array}\end{array}$ & 4222 & 24 & 67 & 49 & & & \\
\hline
\end{tabular}

Table 2 Results from both the Rotterdam and London cohorts

\begin{tabular}{lccccc}
\hline \multirow{2}{*}{ Setting } & \multicolumn{2}{c}{ Rotterdam cohort } & & \multicolumn{2}{c}{ London cohort } \\
\cline { 2 - 3 } \cline { 5 - 6 } & \multicolumn{3}{c}{$\begin{array}{c}\text { Percentage } \\
(\%)\end{array}$} & & \multicolumn{2}{c}{$\begin{array}{c}\text { Percentage } \\
\text { Number }\end{array}$} & $(\%)$ \\
\hline Few Inner, Soft Inner & 8 & 11.9 & & 10.7 \\
Few Inner & 5 & 7.5 & & 5.4 \\
Few Outer & 52 & 77.6 & & 47 & 83.9 \\
Soft Inner & 1 & 1.5 & & 0.0 \\
Few Inner, Soft Outer & 1 & 1.5 & & 0 & 0.0 \\
Total & 67 & 100.0 & 56 & 100.0 \\
\hline
\end{tabular}

expert, following which sensitivity and specificity were derived. The intention in this study was for the software to be eventually used by someone with basic training rather than someone with advanced ophthalmological training (eg, consultant ophthalmologist), and thus we used a trained medical student to analyse the data.

It is also foreseeable that the use of this software and its automated algorithm would tremendously facilitate and expedite the mass screening of drusen formation in the general population by enabling an efficient and accurate analysis and screening of drusen formation. This is achieved by RIALAB being able to analyse drusen relatively quickly - each photograph took about $60 \mathrm{~s}$ to analyse with the processing capability of a personal notebook computer.

The second outcome of this study was that there was a clear default setting (the 'Few Outer' drusen setting), which could be used with this software and algorithm. The 'Few Outer' setting was deemed to be the best setting in $77.6 \%$ of patients in the Rotterdam Elderly Study and $83.9 \%$ in the hospital-based study. Of the remainder, about half of them would be covered by the next best 'Few Inner, Soft Inner' setting. This is again of practical importance, as this means that with just the default setting, about four out of five photographs would be able to be analysed accurately, with the clinician easily making a switch of the settings for those patients whose drusen do not neatly fit into the default setting.

By having a default setting, graders would be able to fully automate their workflow by running the retina 

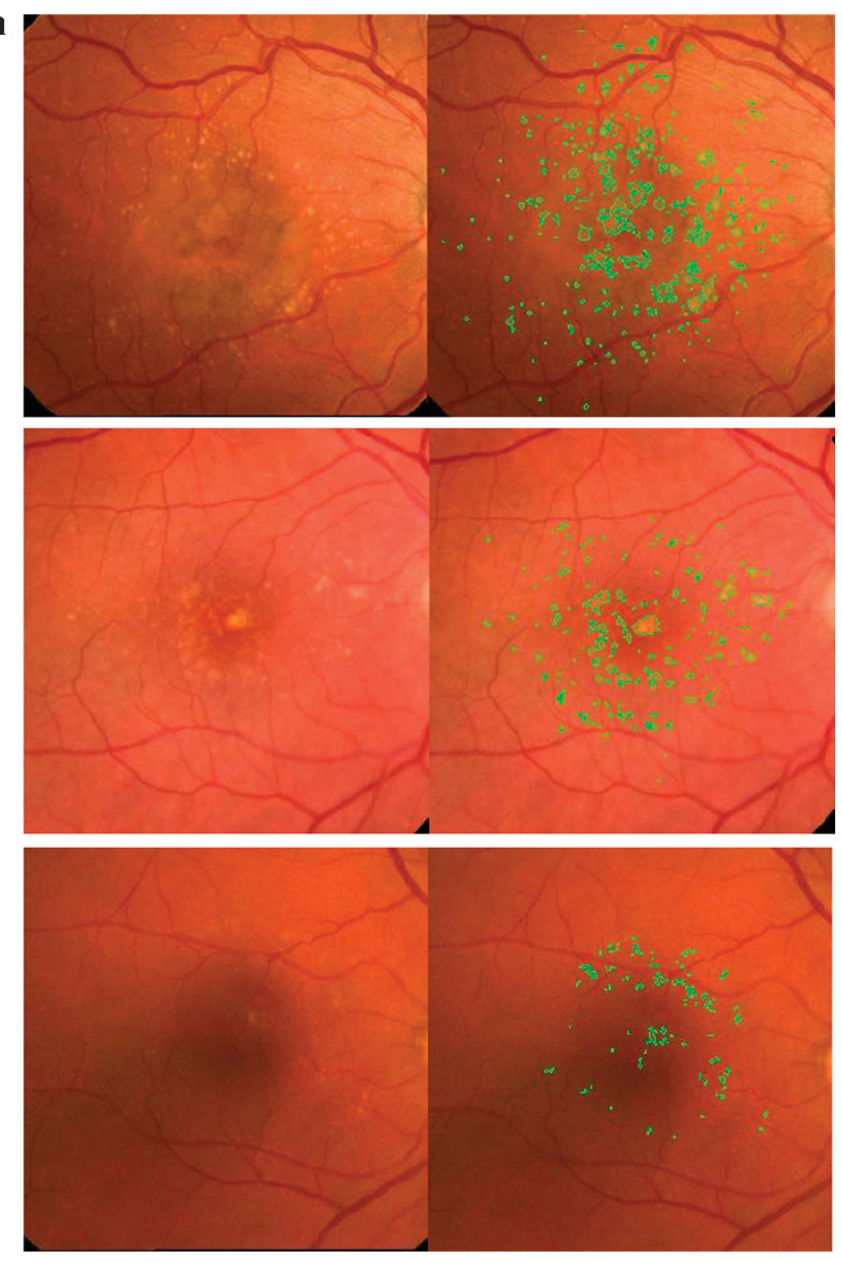

b

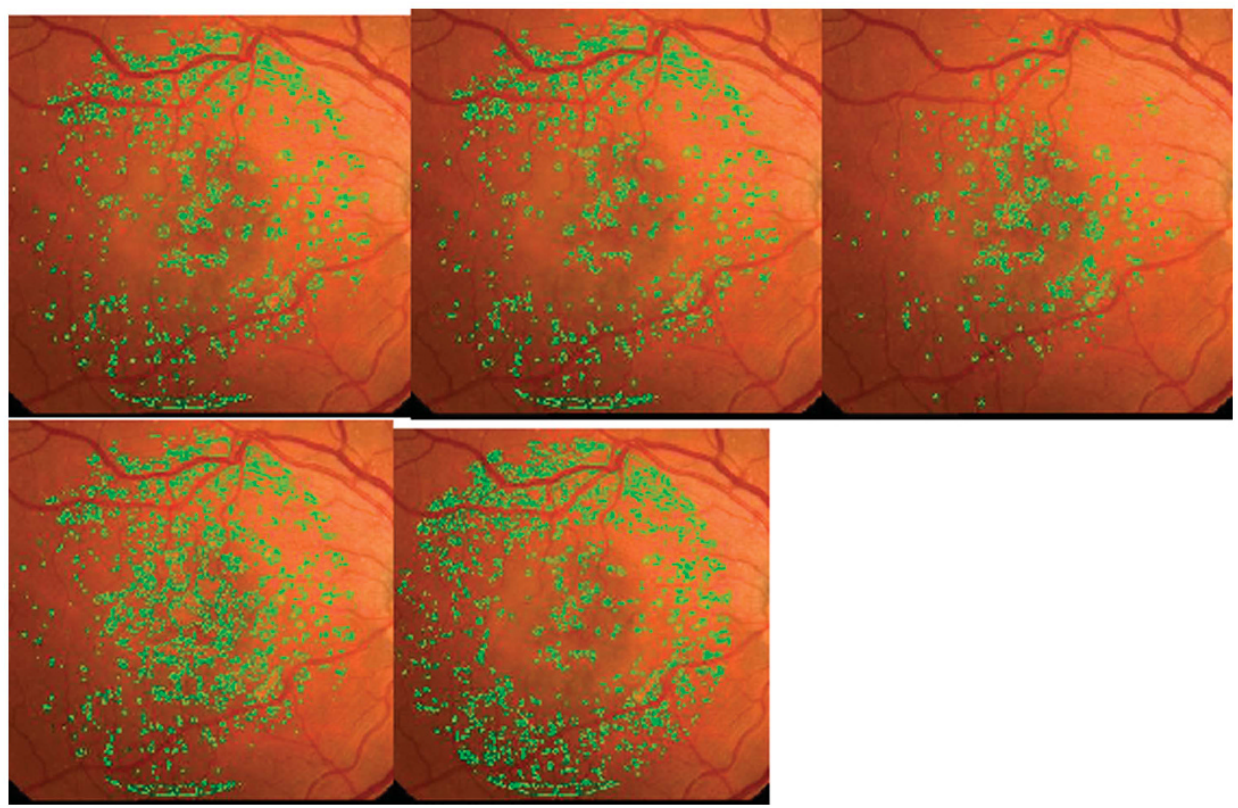

Figure 3 (a) A side-by-side comparison of cropped fundus photographs of eyes in three separate patients, and the same photographs with drusen delineated as the green areas by RIALAB. (b) A comparison of the possible outputs from the different combinations of settings using the software. In this example, option 3 (Few Outer) was deemed to be the best output. 

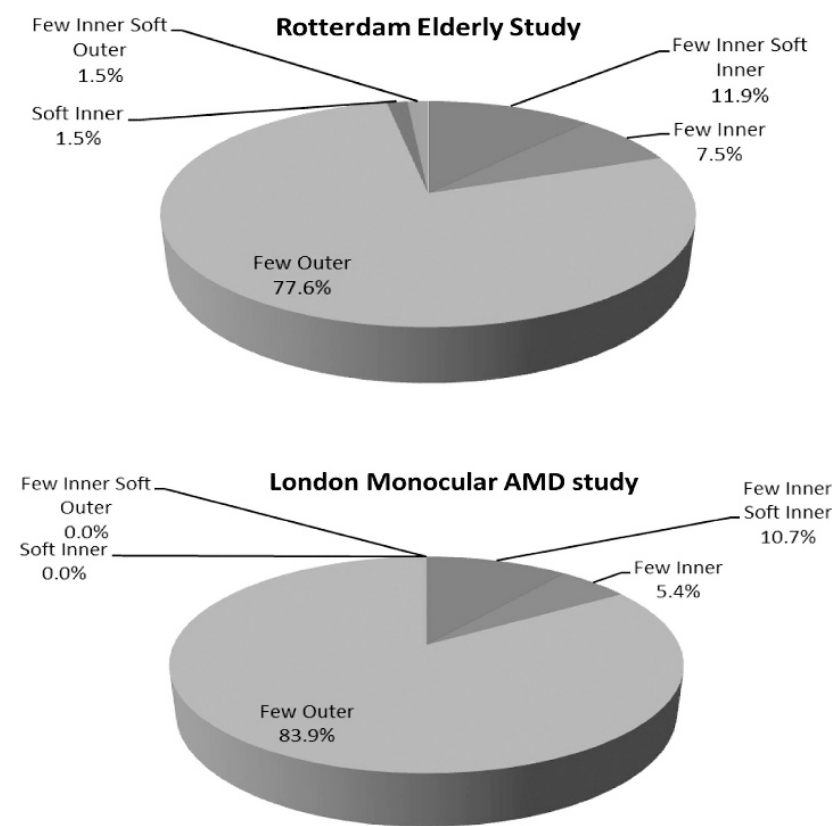

Figure 4 Pie chart showing graphically the distribution of the various settings for RIALAB for the Rotterdam Elderly Study and the London AMD cohort.

photographs through the default setting of 'Few Outer', thus achieving a highly accurate result about $80 \%$ of the time. By quickly scanning through the images pre- and post-analysis, images which do not fit neatly within the default settings could be selected and rerun under the next best setting of 'Few Inner, Soft Inner'. The implementation of a default setting would greatly enhance the workflow of the software by eliminating one more rate-limiting step in the analysis of the software.

Ultimately, this study represents an initial study and a proof of concept of the use of automated software to analyse drusen efficiently and to present an efficient pathway for assessment.

\section{Conclusion}

RIALAB is a viable algorithm and software package that can help to detect, quantify, and analyse drusen efficiently. This could potentially have great utility in assisting primary care clinicians in detecting, and monitoring, the progression of dry AMD. This software would also enable more accurate characterisation of drusen size and distribution as a marker for progression of AMD and response to treatment. In addition, we have shown that one combination of settings, namely the 'Few Outer' drusen setting, can be employed as the default setting in both population-based and hospital-based studies, with fine-tuning only needed in the minority of cases where it is not accurate, thus helping to speed up the workflow even more.
Apart from its potential use in screening, we envisage further research uses for this software in research.

Possible directions in the future could include using it to analyse the prognostic significance the characteristics of drusen, whether it is its distribution in different anatomical regions, size, or by the hardness of its borders. Further collaborations with the Rotterdam Elderly Study regarding its uses in analysis and predicting prognosis are already under way.

\section{Summary}

What was known before

- Manual drusen quantification is a time-intensive process with poor accuracy.

What this study adds

- Computer software can perform automatic drusen quantification, which is more efficient.

- It is possible to configure defaults for the software to further improve throughput, as certain drusen phenotypes more commonly occur, as shown in this paper.

- The use of automatic drusen-quantifying software has numerous advantages, including allowing monitoring of drusen as biomarkers for longitudinal studies and also to expedite mass screening of the population.

\section{Conflict of interest}

The authors declare no conflict of interest. 


\section{References}

1 Age-Related Eye Disease Study Research Group. A randomized, placebo-controlled, clinical trial of high-dose supplementation with vitamins $\mathrm{C}$ and $\mathrm{E}$, beta carotene, and zinc for age-related macular degeneration and vision loss: AREDS report no. 8. Arch Ophthalmol 2001; 119(10): 1417-1436.

2 Klaver CC, Assink JJ, van Leeuwen R, Wolfs RC, Vingerling $\mathrm{JR}$, Stijnen $\mathrm{T}$ et al. Incidence and progression rates of age-related maculopathy: the Rotterdam Study. Invest Ophthalmol Vis Sci 2001; 42(10): 2237-2241.

3 Peli E, Lahav M. Drusen measurement from fundus photographs using computer image analysis. Ophthalmology 1986; 93(12): 1575-1580.

4 Shin DS, Javornik NB, Berger JW. Computer-assisted, interactive fundus image processing for macular drusen quantitation. Ophthalmology 1999; 106(6): 1119-1125.

5 Quellec G, Russell SR, Scheetz TE, Stone EM, Abràmoff MD. Computational quantification of complex fundus phenotypes in age-related macular degeneration and Stargardt disease. Invest Ophthalmol Vis Sci 2011; 52(6): 2976-2981.

6 Schlanitz FG, Ahlers C, Sacu S, Schütze C, Rodriguez M, Schriefl S et al. Performance of drusen detection by spectraldomain optical coherence tomography. Invest Ophthalmol Vis Sci 2010; 51(12): 6715-6721.

7 Pearce E, Ong BB, Brennan N, Smith T, Sivaprasad S, Chong V. Retinal Sensitivity Is Reduced With The Increase Of Drusen Load In Fellow Eye Of Patients With Neovascular Age-related Macular Degeneration. 2012 Annual Meeting, The Association for Research in Vision and Ophthalmology (ARVO) 2012. Abstract available at http:/ / www.abstractsonline.com/Plan/ViewAbstract. aspx?sKey = f59cf793-035b-4cab-af07-e71447362abf\&cKey $=$ fbefffe0-e2e4-4aee-a5dc-9235a904ec34\&mKey $=\%$ 7bF0FCE029-9BF8-4E7C-B48E-9FF7711D4A0E\%7d.

8 World Medical Association. Declaration of Helsinki-Ethical Principles for Medical Research Involving Human Subjects. Sixth Revision, World Medical Association: Ferney-Voltaire, France, 2008.

9 Lee N. RIALAB Documentation Manual. [electronic copy] Undated.

10 Smith RT, Chan JK, Nagasaki T, Ahmad UF, Barbazetto I, Sparrow J et al. Automated detection of macular drusen using geometric background leveling and threshold selection. Arch Ophthalmol 2005; 123(2): 200-206.

11 Otsu N. A threshold selection method from gray-level histograms. IEEE Trans Syst Man Cybern 1979; 9: $62-66$.

12 Hofman A, Breteler MM, van Duijn CM, Janssen HL, Krestin GP, Kuipers EJ et al. The Rotterdam Study: 2010 objectives and design update. Eur J Epidemiol 2009; 24(9): 553-572.

13 Hofman A, Grobbee DE, de Jong PT, van den Ouweland FA. Determinants of disease and disability in the elderly: the Rotterdam Elderly Study. Eur J Epidemiol 1991; 7(4): 403-422.

14 Erasmus MC Department of Epidemiology. The Rotterdam Study 2011. http:/ / www.epib.nl/research/ergo.htm. Accessed: 22 March 2011.

15 Rapantzikos K, Zervakis M, Balas K. Detection and segmentation of drusen deposits on human retina: potential in the diagnosis of age-related macular degeneration. Med Image Anal 2003; 7(1): 95-108. 